\title{
Sunflowers in Lattices
}

\author{
Geoffrey McKenna \\ Department of Mathematics \\ The George Washington University \\ Washington, D.C. 20052, USA \\ gmckenna@gwu.edu
}

Submitted: May 24, 2004; Accepted: Nov 8, 2005; Published: Nov 29, 2005

Keywords: sunflower; lattice; geometric lattice; distributive lattice; divisor lattice

\begin{abstract}
A Sunflower is a subset $S$ of a lattice, with the property that the meet of any two elements in $S$ coincides with the meet of all of $S$. The Sunflower Lemma of Erdös and Rado [2] asserts that a set of size at least $1+k !(t-1)^{k}$ of elements of rank $k$ in a Boolean Lattice contains a sunflower of size $t$. We develop counterparts of the Sunflower Lemma for distributive lattices, graphic matroids, and matroids representable over a fixed finite field. We also show that there is no counterpart for arbitrary matroids.
\end{abstract}

\section{Introduction}

Erdös' Sunflower Lemma, originally presented in [2], has been called "one of the most beautiful results in extremal set theory" ([3], p. 79). In this note we offer a modest generalization. First, a few standard definitions. Let $m$ be a natural number, and denote by $[m]$ the set of integers $\{1,2, \ldots m\}$. A family $F$ is simply a collection of subsets of the ground set $[m] ; F$ is said to be $k$-uniform if each of its members has $k$ elements. A sunflower $S$ of size $t$ is a family $\left\{A_{1}, A_{2}, \ldots A_{t}\right\}$ with the property that every pair of distinct sets in $S$ have the same intersection, i.e., $A_{i} \cap A_{j}=A_{r} \cap A_{q}$ for distinct pairs of distinct subscripts $i, j$ and $r, q$. Denote by the core of $S$ the set $Y:=\bigcap A_{j}$, so $Y=A_{i} \cap A_{j}$ for each pair of distinct $i, j$. The $t$ sets $A_{i} \backslash Y$ are required to be nonempty and distinct, and are called the petals of $S$. Here is the original lemma ([3], p. 79-80); we reproduce the short proof to make the presentation more self-contained.

Lemma 1.1. The Sunflower Lemma: Let $F$ be a k-uniform family of sets. If $|F|>$ $k !(t-1)^{k}$, then $F$ contains a sunflower with $t$ petals.

Proof. Proceed by induction on $k$. If $k=1$ then $F$ has, by assumption, more than $t-1$ petals, so it has a sunflower with $t$ petals and an empty core. Let $T$ be a maximal family 
of pairwise disjoint members of $F$. If $|T| \geq t$, then $T$ itself is the desired sunflower. Otherwise $Z:=\bigcup_{X \in T} X$ is a set of size at most $(t-1) k$, which, by maximality, meets every member of $F$. By the pigeonhole principal, some element $z \in Z$ must lie in at least $\left.|F| /|Z|>k !(t-1)^{k} /(k(t-1))=(k-1) !\right)(t-1)^{k-1}$ elements of $F$. Let $F_{z}$ denote the subfamily of $F$ consisting of those members of $F$ that contain $z$. Define $G_{z}:=\{A \backslash z: A \in$ $\left.F_{z}\right\}$. Then $G_{z}$ is $(k-1)$-uniform, and, by induction, contains a sunflower of the required size.

We propose to repeat this argument, with as little variation as possible, in a few other settings. In each new setting considered here, we require an explanatory preamble indicating what a pertinent sunflower is, followed by the above proof, mutatis mutandis. Paraphrasing the above proof, here is an outline of the argument we plan to follow for each setting:

(i) Suppose that $F$ is a $k$-uniform family of size at least $k !(t-1)^{k}$.

(ii) Seek a maximum sunflower $S$ in $F$ with an empty core. If it is of size at least $t$, we're done.

(iii) Otherwise, the join $Z$ of all the members of $S$ is of low rank, and meets all members of $F$.

(iv) It follows that $Z$ dominates a rank-1 object $z$ meeting a relatively large percentage of the members of $F$. Careful removal of $z$ from these members of $F$ finishes the inductive argument.

Any family of size 1 or 2 is a sunflower, so the content of the lemma applies when $t>2$, which is the case to which we can reasonably confine our attention.

\section{The Divisor Lattice}

We start with the simplest, most modest, and most concrete of generalizations. Let $M$ be a large unspecified integer. Following Stanley [6], we denote by $D_{M}$ the set of divisors of $M$, ordered by the relation $a \mid b \Longleftrightarrow a \leq b$. By an antichain we then mean a set of divisors of $M$, none of which divides another. By the $\operatorname{rank} r(d)$ of $d \in D_{M}$ is meant the number of prime factors of $d$, counting multiplicity. For example, $r(12)=r(2 \times 2 \times 3)=3$. Call a set $S \subseteq D_{M}$ k-uniform if each element of $S$ has rank $k$. By a sunflower we mean a set $S \subseteq D_{M}$, with the property that the Greatest Common Divisor (GCD) of any pair is the GCD of the whole collection. Denote the GCD of all the members of $S$ by $Y$, which we call the core of $S$.

Lemma 2.1. The Sunflower Lemma: Let $F$ be a k-uniform family of divisors of $M$. If $|F|>k !(t-1)^{k}$, then $F$ contains a sunflower with $t$ petals. 
Proof. Proceed by induction on $k$. For the base case, if $k=1$, then $F$ itself is a sunflower consisting of $|F| \geq t$ distinct prime numbers. Assume $F$ to be $k$-uniform for $k>1$. Let $S$ be a maximal sunflower with empty core. If $|S| \geq t$ we're done, so assume $|S|<t$. Observe that the least common multiple $L$ of the members of $S$ is a divisor of $M$, and has rank at most $k(t-1)$. By the maximality of $S, L$ has at least one prime factor in common with each member of $F$. Therefore, by the pigeonhole principle, some prime factor $z$ of $L$ occurs in at least $|F| /|Z| \geq(k !)(t-1)^{k} /(k(t-1))$ elements of $F$. Let $F_{z}$ denote the subfamily of $F$ consisting of those members of $F$ divisible by $z$. Define $G_{z}:=\left\{A / z: A \in F_{z}\right\}$. Then $G_{z}$ is $(k-1)$-uniform, and, by induction, contains a sunflower of the required size.

Remark: It is essential to this argument that we can pass from $F_{z}$ to $G_{z}$, in this case by division. Analogues of division exist for distributive lattices as we show next, but are more problematic for general lattices. After considering some "non-examples", we arrive at a form of the Sunflower Lemma for Graphic matroids, and for each class of matroids characterized by representability over a particular Galois field.

\section{$3 \quad$ Distributive Lattices}

This case generalizes the preceding, since divisor lattices are distributive. Recall that a lattice $L$ is distributive if it obeys the distributive laws, i.e., given $a, b, c \in L$, we have $(a \wedge b) \vee c=(a \vee c) \wedge(a \vee b)$ and $(a \vee b) \wedge c=(a \wedge c) \vee(a \wedge b)$. As will prove handy, the Fundamental Theorem of Distributive Lattices ( [1], p. 33; [6], p. 106) states that each distributive lattice is isomorphic to the lattice of ideals of the poset of its join-irreducible elements. To simplify the discussion we confine our attention to finite lattices. In a distributive lattice, the rank of an element $a$ is the number of join-irreducibles that it dominates. All rank-1 elements of $L$ are join-irreducible. There may, as in the case of the divisor lattice, be others. Observe that if $L$ is any lattice (distributive or not), $a, b \in L$, and $a \wedge b \neq \hat{0}$, then there is an atom of $L$ lying beneath both $a$ and $b$ - any minimal nonzero element will do.

As elsewhere, by a sunflower in $L$ we mean a set of elements of $L$, any two of which have the same meet.

Theorem 3.1. The Sunflower Lemma: Let $F$ be a set of elements of rank $k$ in a distributive lattice L. If $|F|>k !(t-1)^{k}$, then $F$ contains a sunflower with $t$ petals.

Proof. Let $S$ be a maximal subcollection of $F$, any two of which meet at the zero element of $L$. We can assume that $|S|<t$, since otherwise we're done. Observe that the join $Z$ of all the members of $S$ is an element of rank at most $k(t-1)$. By maximality, $\operatorname{rank}(Z \wedge a)>0$ for each $a$ in $F$. Since every atom $x$ for which $Z \geq x$ is join-irreducible, there are at most $k(t-1)$ atoms dominated by $Z$. Denote by $A$ the set of atoms of $L$ dominated by $Z$. By the preceding statement, each element of $F$ meets (i.e., has nonzero meet with) at least one member of $A$. So, by the pigeonhole principle, some member $z$ of $A$ meets at least $|F| /|A| \geq(k !)(t-1)^{k} /(k(t-1))$ elements of $F$. Again let $F_{z}$ be the subfamily of $F$ consisting of those elements that dominate $z$. Let $Q$ be the set of join-irreducibles 
of $L$, and let $M$ be the distributive lattice of ideals of the poset $Q \backslash z$. We define a set homomorphism $g: 2^{Q} \rightarrow 2^{Q \backslash z}$ defined by $g(X):=X \backslash x$, so $g$ fixes those subsets of $Q$ that omit $z$. Consider the lattice homomorphism $f: L \rightarrow M$ induced by $g$, i.e., $f$ is the restriction of $g$ to the ideals of $Q$. Observe that the restriction of $f$ to $F_{z}$ is an injection ( here we identify $L$ with the lattice of ideals of $Q$.) Denote the image $f\left(F_{z}\right)$ by $G_{z}$. Then $G_{z}$ is a $(k-1)$-uniform set of elements in the distributive lattice of ideals of $Q \backslash z$, so by induction it contains a sunflower $Y$ of the required size. But then $f^{-1}(Y)$ is the sunflower we seek in $L$.

The reader may reasonably object that our careful selection of an atom dominated by many members of $F$ is essentially unnecessary; any join-irreducible dominated by a large number of members of $F$ would do. The method given here was chosen to emphasize the similarities with the original, set-theoretic form of the Sunflower Lemma.

\section{Two Non-Examples}

Non-example 1: We construct a lattice of rank 3 with the property that, even though the number of elements of rank 2 is as large as we please, there are no sunflowers of size greater than 2 to be found among the elements of rank 2. Here's how: Let $K_{n}$ be the complete graph on $n$ vertices. The atoms of our lattice are the edges of the graph, and the rank-2 elements of the lattice are the vertices of the graph. The join of two edges is their common vertex where applicable, and the $\hat{1}$ element otherwise. Note that any two vertices share a common atom, and that no two distinct pairs of vertices dominate the same edge.

Non-example 2: Let $m$ be a large prime power, and consider the finite projective plane $P G(2, m)$ over the Galois field $G F(m) . P G(2, m)$ contains $p:=m^{2}+m+1$ points. Pick nine lines at random. Now, if the Sunflower Lemma applied here, then we would be assured that three of these lines meet at a point, because lines have rank $k=2$, and $9>2 !(3-1)^{2}=8$. To phrase this in the language of probability, the Sunflower lemma asserts that the probability that some three of these nine lines form a Sunflower is 1 . Since the intersection of any two distinct lines is a point, there must then be some point $x$ common to all three lines. Fix a point $x$ of the geometry. Now, each point is incident to $m+1$ lines, so the probability that three or more points contain $x$ is $\sum_{k=3}^{9}\left(\begin{array}{c}m+1 \\ k\end{array}\right)\left(\begin{array}{c}p-(m+1) \\ 9-k\end{array}\right) /\left(\begin{array}{c}p \\ 9\end{array}\right)<2^{9}\left(\begin{array}{c}m+1 \\ 3\end{array}\right)\left(\begin{array}{c}m^{2} \\ 6\end{array}\right) /\left(\begin{array}{c}m^{2} \\ 9\end{array}\right)<c m^{-3}$ for some positive constant $c$. But then the probability that any point appears in three or more lines is less than $p c m^{-3}=\theta(1 / m)$.

From the standpoint of collecting sunflowers, the second Non-example is particularly discouraging, because projective planes possess so many nice structural properties. Projective planes are both binomial posets and geometric lattices. What went wrong?

The answer is: In each of our Non-examples, the number of atoms is large relative to the rank of the lattice. So, if we confine our attention to classes of geometric, and perhaps even submodular, lattices in which the number of atoms is bounded by a function that 
grows relatively slowly with the rank of the lattice, perhaps we can improve our prospects for success. This tack we now take.

\section{Graphic Matroids}

Let $G=(V, E)$ be a graph. Recall that the cycle matroid $M(G)$ of $G$ is described by the rule: a set of edges $S \subseteq E$ is independent iff it is acyclic. For more details on graphic matroids see [5]. As is true of any matroid, the lattice of flats of a graphic matroid is a geometric lattice ( [1], chapters II.3 and VII.3), i.e., semimodular and graded. In the language of graph theory, a flat in a graphic matroid $M(G)$ is any set of edges that can be obtained by the following recipe: First, pick a subforest $F_{0}$ of $G$. Second, adjoin any remaining edges of $G$ connecting two vertices incident to $F_{0}$. The resulting collection $F_{1}$ of edges is said to be a flat of $M(G) ; F_{0}$ is said to be a basis of this flat. The number of edges in the basis $F_{0}$ is the rank of the flat $F_{1}$ in $\mathcal{L}(M(G))$, the lattice of flats of the matroid $M(G)$. To avoid technicalities involving parallel edges, we use the word atom instead of edge to denote a rank-1 flat. Note that we are not directly studying the graph $G$ or even its cycle matroid $M(G)$, but rather the lattice of flats $\mathcal{L}(M(G))$.

Lemma 5.1. Let $G$ be a graph, $M(G)$ its cycle matroid, and $\mathcal{L}(M(G))$ its lattice of flats. The number of atoms dominated by a flat of rank $k$ in the lattice of flats of a graphical matroid is at most $\left(\begin{array}{c}k+1 \\ 2\end{array}\right)$.

Proof. Let $F_{1}$ be a flat spanned by the basis $F_{0}$, where the latter constitutes a forest of $k$ edges. We can proceed by induction on the number of components of $F_{0}$. ( It is true that all bases of $F_{1}$ have the same number of components, a fact not required for the proof). If $F_{0}$ consists of a single component, then it is a tree with $k$ edges. It follows that the set $X$ of vertices of $G$ incident to $F_{0}$ has $k+1$ elements. The greatest possible number of edges in $G$ incident to $X$ occurs if the induced subgraph of $G$ on $X$ is complete, in which case we get $\left(\begin{array}{c}k+1 \\ 2\end{array}\right)$ edges dominated by $F_{1}$. As each rank-1 flat of $M(G)$ has a basis consisting of a single edge, there are at most $\left(\begin{array}{c}k+1 \\ 2\end{array}\right)$ atoms of $M(G)$ dominated by $F_{1}$. For the inductive step, the important arithmetic observation is $\left(\begin{array}{c}k \\ 2\end{array}\right) \geq\left(\begin{array}{c}i \\ 2\end{array}\right)+\left(\begin{array}{c}k-i \\ 2\end{array}\right)$ for each $i$ between 0 and $k$.

In the case of a Graphic Matroid $M(G)$, by a $k$-uniform family we mean a family of flats of rank $k$ in $\mathcal{L}(M(G))$. The rank of a flat $X$ is the cardinality of any basis, (i.e., maximal independent subset), of $X$. Here a sunflower is a $k$-uniform family $F$ of flats, with the property that any two members of $F$ have the same intersection. Note that the meet of a pair of flats in $\mathcal{L}(M(G))$ is simply their intersection. For our purposes, the single most interesting fact about the lattice of flats is its submodularity: Given a collection $\left\{X_{i}\right\}$ of flats, where the basis of $X_{i}$ is $B_{i}$, the rank of $\vee X_{i}=\operatorname{rank}\left(\cup B_{i}\right) \leq$ $\left|\cup B_{i}\right| \leq \sum B_{i}=\sum \operatorname{rank}\left(X_{i}\right)$.

We further require the following technical lemma from the theory of matroids ( [5], Corollary 3.3.3, p. 118, also [1], Proposition 6.35.ii, p. 286): 
Lemma 5.2. Let $M$ be matroid, $\mathcal{L}(M)$ its lattice of flats, and $X$ a flat of $M$. Then the sublattice of flats containing $X$ is isomorphic to the lattice of flats of the contraction $M / X$. In symbols, if $[X, 1]$ denotes the interval of flats from $X$ to $M$, then $[X, 1] \cong \mathcal{L}(M / X)$.

The proximate case of interest is that where $M$ is the cycle matroid $M(G)$ of a graph $G$, and where $X$ is a rank-1 flat in $M(G)$.

Theorem 5.3. Sunflower Lemma for Graphic Matroids: Let $t>2$, and let $F$ be a $k$ uniform family of size at least $\left[\prod_{1 \leq j \leq k}(\underset{2}{(t-1) j+1})\right]+1$ in the graphic matroid $M(G)$ for some graph $G$. Then there is a sunflower of size $t$ in $F$.

Proof. We proceed by induction on $k$. For the base case, if $k=1$, then $F$ itself is a sunflower consisting of $|F| \geq t$ pairwise disjoint flats. Assume $F$ to be $k$-uniform for $k>1$. Let $S$ be a maximal sunflower with empty core, i.e., a set of pairwise disjoint flats. If $|S| \geq t$ we're done, so assume $|S|<t$. Observe that the join $Z$ of all the members of $S$ is a flat of rank at most $(t-1) k$ by submodularity. By the maximality of $S, \operatorname{rank}(Z \cap Y)>0$ for each flat $Y$ in $F$. Since the rank of $Z$ is at most $(t-1) k$, by Lemma 5.1 there are at most $\left(\begin{array}{c}(t-1) k+1 \\ 2\end{array}\right)$ atoms of $M(G)$ dominated by $Z$. Since every member of $F$ dominates at least one of these atoms, by the pigeonhole principal there is an atom $z$ in $Z$ lying below at least $|F| / \operatorname{rank}(Z) \geq\left[\frac{\prod_{1 \leq j \leq k}\left(\begin{array}{l}(t-1) j+1 \\ 2\end{array}\right)}{\left(\begin{array}{c}(t-1) k+1 \\ 2\end{array}\right)}\right]+1$ members of $F$. Denote by $F_{z}$ the collection of members of $F$ lying above the atom $z$. Denote by $G_{z}$ the collection $\left\{A / z: A \in F_{z}\right\}$, where $A / z$ denotes matroid contraction. Now, $G_{z}$ isn't a subset of $\mathcal{L}(M(G))$, but it is a $k-1$-uniform family in the lattice of flats of the graphic matroid $M(G) / z$. So, by induction, $G_{z}$ contains a sunflower $T$ of the required size. Since the function $f: F_{z} \rightarrow G_{z}$ given by $f(A)=A / z$ is, by the prior Lemma, injective, $f^{-1}(T)$ is the required sunflower in $F$.

Remark: So far we have used the same proof three times, but the semantic content of "removal of an atom" varies enormously from case to case, viz., remove an element from a set, or divide by a prime factor, or, as here, contract a matroid by a rank-1 flat. These cases are unified near the end of the note, at some cost in concreteness.

\section{Matroids representable over a fixed $q$-element field}

The standard designation for matroids representable over a $q$-element field is $L(q)$. A distinguishing trait of these matroids is that lines that contain $q+2$ or more points are forbidden, although lines that containt $q+1$ points are possible. The class $L(q)$ adheres to other restrictions that elude our present interest, so we will confine the discussion to the somewhat larger class $U(q)$ of matroids defined by the rule that $q+2$ - point lines are forbidden. The classes $L(q)$ and $U(q)$ coincide for $q=2$. For larger prime powers, $U(q)$ is strictly bigger [4]. Naturally, any theorem we can construct for the larger class will work for the smaller class. An additional advantage of $U(q)$ is that $q$ need not be a prime power. The following lemma appears in stronger, but more technical form in [4], Theorem 4.3, p.32: 
Lemma 6.1. Let $M$ be a matroid of rank $k$ in $U(q)$. Then the lattice $L(M)$ of flats of $M$ has at most $\frac{q^{k}-1}{q-1}$ atoms.

Proof. If $M$ has rank 2, then it has at most $q+1$ atoms by the definition of $U(q)$. We proceed by induction on the rank of $M$, which we suppose to be at least 3 . Let $x$ be any atom of $M$. The lattice interval of flats lying between $x$ and 1 (i.e., the set of flats of $M$ containing $x$, ordered by inclusion and viewed as an interval of $\mathcal{L}(M)$ ), is a lattice of rank $k-1$ in $U(q)$, so by induction it has at most $\frac{q^{k-1}-1}{q-1}$ atoms, each of which is simply a rank-2 flat of $M$ covering $x$. Each flat $y$ covering $x$ covers at most $k$ other atoms of $M$, again by the definition of $U(q)$. Let $z$ be some other atom of $M$. By submodularity, $\operatorname{rank}(x \vee z)=2$, so there is some flat $y$ of rank 2 dominating both $x$ and $z$. Consider the subgraph of the Hasse diagram of $L(M)$ induced on the vertices of rank 1 or 2. Every atom can be of distance at most 2 from $x$, but then there can be at most $\frac{q\left(q^{k-1}-1\right)}{q-1}$ other atoms of $M$. But $1+\frac{q\left(q^{k-1}-1\right)}{q-1}=\frac{q^{k}-1}{q-1}$.

Theorem 6.2. Sunflower Lemma for Matroids in $U(q)$ : Let $t>2$, and let $F$ be a $k$ uniform family of size at least $\prod_{1 \leq j \leq k} \frac{q^{j t}-1}{q-1}+1$ in the matroid $M(G) \in U(q)$. Then there is a sunflower of size $t$ in $F$.

Proof. Repeat the proof for graphical matroids, changing only the bound on the number of atoms dominated by a flat of rank $j$.

\section{A Generalization and an Application}

A key feature of all proofs in this note is reliance on the fact that there aren't too many atoms in play. We may formalize this reliance with a definition: A class $C$ of lattices will be deemed oligatomic if it follows these rules:

(i) Every interval of a member of $C$ is in $C$.

(ii) Every member of $C$ is submodular.

(iii) There is a bound $r+1$ on the number of atoms in a rank-2 member of $C$.

The significance of the number "2" in item(iii) is that, by the same argument as given in Lemma 6.1, there can then be at most $\frac{r^{k}-1}{r-1}$ atoms in a member of $C$ of rank $k$. We note that the classes of lattices mentioned in each section heading of this note are oligatomic. Here is the generalization to which the section heading refers:

Theorem 7.1. A generic Sunflower Lemma: Let $C$ be an oligatomic class of lattices, and let $r_{k}$ denote the maximum number of atoms occurring in a member of $C$ of rank $k$. Let $k$ and $t$ be integers. Suppose $L \in C$, and suppose $F \subset L$ to be a $k$-uniform family of size greater than $\prod_{1 \leq j \leq k} r_{j(t-1)}$. Then $F$ contains a sunflower of size $t$.

Proof. Same proof. 
Now for the application. Consider a formal meet $f:=X \wedge Y$. Given a sunflower $S$ in some lattice $L$, the evaluation map $f: S^{(2)} \rightarrow L$ is, by the definition of a sunflower, a constant map. Now consider a three-term formal meet $g:=X \wedge Y \wedge Z$. We can rewrite this as $g=X \wedge Y \wedge Z=(X \wedge Y \wedge Z) \wedge(X \wedge Y \wedge Z)=(X \wedge Y) \wedge(Y \wedge Z) \wedge(X \wedge Z)$. But then, evaluation of $g$ at any element of $S^{(3)}$ yields the same answer $g(x, y, z)=f(x, y)$. Likewise, if we construct more elaborate formal polynomials of the form $p:=\bigvee_{i \in I} \bigwedge_{u \in S^{\left(r_{i}\right)}}$, as long as every meet occuring in this polynomial has at least $r_{i} \geq 2$ terms for all $i$, evaluation of the polynomial in $S$ yields the same answer $f(x, y)$. [ There is a technicality to consider - what happens when there are monomials in $p$ containing more terms than $|S|$ ? One solution is to say, if $p$ can be evaluated at all assigning distinct values to the variables in each of its monomials, then $p$ evaluates to the familiar value $f(x, y)]$. Call a polynomial of this form, in which each monomial $\bigwedge_{u \in S^{\left(r_{i}\right)}}$ has at least two terms, admissible.

The upshot is that a sunflower is a species of algebraic variety. By this we mean that, given a finite set $E$ of admissible polynomials and a natural number $k$, for all sufficiently large values of $t$, and given any sufficiently large $k$-uniform family $F$, we may find a subfamily $S \subset F$ of size $t$ such that all polynomials in $E$ evaluated in $S$ have the same value, i.e., no matter which polynomial $p$ we pick from $E$, and no matter which distinct elements of $S$ we assign to the terms of each monomial in $p$, we get the same value. For $S$, we may take any sunflower of size at least as large as the largest monomial appearing in $E$; this is the reason for the stipulation that $t$ be "sufficiently large".

\section{Concluding Remarks}

The matroid-theoretic version of the lemma given here guarantees a sunflower of size about $\theta \frac{\log _{q}(|F|)}{k^{2}}$, as opposed to the putative lower bound of about $\theta \log (|F|)$ obtained in both the set-theoretic and distributive cases. For a particular class $\mathcal{C}$ of submodular lattices, one may define a Ramsey-type function $f(\mathcal{C}, k, t)$ indicating the maximum over $L \in \mathcal{C}$ of the maximum size of a $k$-uniform family $F$ of elements member of $L$ without a sunflower $S$ of size $t$ contained in $F$. The point of such a function is to identify classes in which relatively large sunflowers are guaranteed to exist. Such classes are distinguished by relatively low values of $f$. Also, $f$ obeys a kind of monotonicity: if $\mathcal{D}$ is a superclass of $\mathcal{C}$, then $f(\mathcal{D}, k, t) \geq f(\mathcal{C}, k, t)$.

Among the classes of non-free matroid treated in this note, Graphic Matroids yield the largest sunflowers. This suggests two possibile directions in which to extend the results presented here: by treating classes of matroid closely related to Graphic Matroids, or by treating Graphic Matroids for specific classes of Graph.

So, here are three concrete cases to consider:

(i) Regular Matroids. These are the matroids representable over any field, and constitute a relatively small superclass of the Graphical Matroids. If we define $\mathcal{R}$ to be the class of Regular Matroids and $\mathcal{G}$ to be the subclass of Graphical Matroids, is it true that $f(\mathcal{D}, k, t)=f(\mathcal{C}, k, t)$ ? 
(ii) Graphic Matroids for sparse graphs and random graphs. The idea here is as follows: If $G$ is acyclic, then its cycle matroid is simply a boolean lattice, in which the original, strongest form of the Sunflower Lemma prevails. Likewise, one would expect $f(\mathcal{C}, k, t)$ to be relatively low for a class $\mathcal{C}$ of graphs in which the number of cycles relatively low. One can't simply tie $f(\mathcal{C}, k, t)$ to the edge density of the graphs in $\mathcal{C}$, because a dense subgraph can yield a large Sunflower-free family. Perhaps an ideal result along these lines would be as follows: Given a random graph $G:=$ $\mathcal{G}(n, p)$, present a concrete upper bound on the likelihood that $M(G)$ contains a family of flats $F$ of size $r$, and such that $F$ contains no Sunflower of size $t$. Such a result would require tools more substantial than those employed here, but Stochastic Sunflower theory seems potentially worthwhile.

(iii) Graphic Matroids for graphs of low chromatic number. Again, it is desirable to use the structure of the graphs under study in $\mathcal{C}$ to bound $f(\mathcal{C}, k, t)$.

Acknowledgements: The author would like to thank Joseph Bonin and Daniel Ullman for their contributions to this note in the areas of grammar, mathematical content, spelling, arithmetic, and presentation.

\section{References}

[1] M. Aigner, Combinatorial Theory, Springer 1979

[2] P. Erdös and R. Rado, Intersection Theorems for Systems of Sets, J. London Math. Soc. 35, 1960, pp. 85-90.

[3] S. Jukna, Extremal Combinatorics, Springer 2001, pp. 79-88.

[4] J. Kung, Extremal matroid theory, in N. Robertson and P. D. Seymour, eds., Graph Structure Theory, Contemporary Mathematics, Volume 147, Amer. Math. Soc., Providence, R. I., 1993, pp. 21-62.

[5] J.G. Oxley,Matroid theory. Oxford University Press, New York 1992

[6] R. Stanley, Enumerative Combinatorics Volume I, Wadsworth\& Brooks/Cole 1986 